\title{
Preference Template of D-Robots
}

\author{
HOU Yuemin ${ }^{1,2, a}{ }^{*}$ and JI Linhong ${ }^{2,3, b}$ \\ ${ }^{1}$ Beijing Information Science and Technology University, P. R. China \\ ${ }^{2}$ Tsinghua University, P. R. China \\ ${ }^{3}$ State Key Laboratory of Tribology, P. R. China \\ a hym01@mails.tsinghua.edu.cn, bjilh@tsinghua.edu.cn
}

Keywords: Computational synthesis, Design robot, Preference, Template.

Abstract. The term of design robot (D-Robot) refers to a finite state computing entity that changes the state of the object through a set of variable programmed actions for the performance of a variety of tasks that need both action-level mental activities of human designers and iterative operational activities. The duties of D-Robots is to take actions to transfer design state based on both rule reasoning and preference. A D-Robot's preference is situated based on its environment and depends on certain psychological characteristics including thinking style, decision making style, level of risk-aversiveness and intention of collaboration. The paper reviews the concept of preference, and proposes a preference template of D-Robots.

\section{Introduction}

Designing includes a series of mental and physical activities including conceiving, analysis, drawing, and computing etc.. Many design automation methods and tools are proposed and developed, but few of them are used in real engineering design. One reason is that to develop such systems costs much more money and time than to develop the products themselves, and in many cases, such systems needs major modification to reuse them for the design of new products. Therefore, toolkits that assist engineers in some design activities rather than to substitute them are practical tools in real design practices of engineering products.

We have proposed the concept of design robot (D-Robot) to achieve specific design tasks [1]. The term of (D-Robot) means a finite state computing entity that changes the state of the object through a set of variable programmed actions for the performance of a variety of tasks that need both action-level mental activities of human designers and iterative operational activities. One characteristics of D-Robot is that it takes actions based on both rules and preference, which distinguishes it from agents and other design automation software tools. Preference is the most difficult part to address a D-Robot. The paper firstly presents a brief review on the concepts of preference and then formulates a preference template of D-Robots.

\section{The Concept of Preference}

The notion of preference varies between philosophy, psychology, economics and social science. Huge amount of literature define and analyze the notion of preference. Here is a philosophical definition of broad preference given by John Broome, a philosophy professor.

$N$ prefers $a$ to $b$ if and only if $N$ is in a mental state that would typically cause $N$ to choose $a$ were $N$ to have a choice between $a$ and $b$ only. a choice between a and b only. We call the mental state a preference for a over $b .[2]$.

Why does $\mathrm{N}$ choose $a$ rather than $b$ ? The choice is based on $N$ 's utility, according to economics. The utility may be measured by the degree of happiness, satisfaction, gratification, enjoyment, or utility they provide (whether real or imagined) [3]. 
In psychology, preferences could be conceived of as an individual's attitude towards a set of objects, typically reflected in an explicit decision-making process [4]. Preferences are context sensitive because preferences depend on goals and goals change over time and across context. Preferences are calculated and the degree of calculation depends on the goals, cognitive constraints, and the experience of the decision maker. Preferences also depend on decision maker's domain knowledge, experience and psychological characters like thinking style, decision making style, degree of risk-aversion, working style and other characters that may influence the product quality and design effectiveness. The preference construction is based on these factors.

Thinking styles. Five different styles of thinking are classified: Synthesis, Idealists, Pragmatists, Analysts and Realists [5].

(1) Synthesis. The Synthesis sees likeness in apparent unlike, sees conflict and form new ideas from that conflict. The strategies of the Synthesis are open argument and confrontation, and ask a question to which they already know the answer in order to get the respondent to open up.

(2) Idealist. The Idealist tries to think holistically, seeks ideal solutions. One of their prime strategies is to "focus on the whole".

(2) Pragmatist. The Pragmatist seeks shortest route to a solution. The main strategy employed by a Pragmatist is quick payoff and short term thinking.

(4) Analyst. The Analyst looks at things logically and is data and fact centered. Their basic strategy is to use methodology and the scientific method.

(5) Realist. The Realist relies on facts and expert opinions and likes to simplify things in to clear and concrete picture, and is interested in concrete results. The main strategy employed by a Realist is that of "Empirical Discovery."

Decision making styles. "The best designs come from not one, but hundreds of well-made decisions. The worst designs arise out of hundreds of poorly-made decisions. All that stands between you and a great design is the quality of your decisions". (Where do they come from? - Jared Spool). Decision making style is a cognitive process which represents the way an individual approaches a problem. There are two key factors in how individuals vary in making decisions: information use and focus (number of solutions explored) [6]. Decision style may assist in predicting decision outcomes [7].

Table 1 Behavioral Reactions According to Rowe and Mason's Decision Styles*[7]

\begin{tabular}{lllll}
\hline $\begin{array}{l}\text { Decision } \\
\text { Style }\end{array}$ & Reaction to & Motivated by & Solves Problems By & $\begin{array}{l}\text { Thinking } \\
\text { Mode }\end{array}$ \\
\hline Analytical & Procedural & Problems & Analysis and Insight & Logical \\
Behavioral & Evading & Acceptance & Feeling and Instinct & Emotional \\
Conceptual & Erratic & Recognition & Intuition and Judgment & Creative \\
Directive & Explosive & $\begin{array}{l}\text { Power and } \\
\text { Status }\end{array}$ & Rules and Policies & Focused \\
& & State & \\
\hline
\end{tabular}

* Rowe \& Boulgarides, 1992, (Relationship between Principals’ Decision Making Styles).

Risk-aversion. Google and Facebook and product design companies such as IDEO have become successful because they take risks that traditional, risk-averse companies are not willing to take [8].

"Risk aversion is a concept in psychology, economics, and finance, based on the behavior of humans (especially consumers and investors) while exposed to uncertainty to attempt to reduce that uncertainty." [9]. If a person's certainty equivalent for alternatives specified in terms of profits is less than/equal to/greater than the expected profit for an alternative, this person is said to be risk averse/risk neural/risk seeking with respect to this alternative. [10].

Risk-aversion models are derived from utility functions. The methods to measure the degree of risk-aversion can be, for example, $\rho \square-u^{\prime \prime}(x) / u^{\prime}(x)$ (the Arrow-Pratt measure of risk-aversion), where $u(x)$ is utility function over any commodity $x, u^{\prime}(x)$ and $u^{\prime \prime}(x)$ represent the first and the second derivative. 
In design, the decision maker's risk attitude can be introduced to express the value of a designed artifact to the enterprise [8]. The degree of risk-aversion of the designer influences the strategy to make changes on existing design.

Working styles. Working styles here refers to the strategies to deal with conflict goals or plans.

Five working styles can be identified [11].

(1) Competing Style: the concerns and the position of the opposition are completely ignored.

(2) Compromising style: both parties are willing to trade some of their needs in order to win concessions from the other side.

(3) Collaborating style: both sides are satisfied.

(4) Accommodating style: both sides are trying to find a solution which truly satisfies the needs of each at the cost to give up just about everything in order to preserve the relationship with the other party.

(5 Avoiding style: the consequences of delay in making a decision will be minimal for both parties so they avoid making a decision at whatever cost. This strategy is often used to delay determining uncertain parameters in design.

Utilities Function. In design, designers make decision based on either reasoning or preference. When the information is completed or almost is completed, decisions can been made based on rule reasoning, game theory or other decision making methods. When alternative solutions are available, decision can be made based on optimization. In other cases, preference based on heuristics are common used methods to make decisions. In all cases, utility function is required.

There are various methods to construct the utility functions. In engineering design, the most common weighted sum method is linear summation of subobjective functions. Other methods include Ranking methods (the different objective functions are ordered by importance), Ratio questioning methods (objective functions are rated by comparing two objectives at a time), Lexicographic methods (the objective functions are arranged in order of importance), Weighted Tchebycheff methods (weighted min-max formulation), Exponential weighted criterion methods $\left(U=\sum_{i=1}^{k}\left(e^{p w_{i}}-1\right) e^{p F_{i}(x)}\right)$, Weighted product methods $\left(U=\prod_{i=1}^{k}\left[F_{i}(x)\right]^{w_{i}}\right.$ ), Goal programming methods (goals $b_{j}$ are specified for each objective function $F_{j}(x)$ ), Bounded objective function methods (the most important objective function is selected as the top objective function and all other objective functions are used to form additional constraints), Physical programming methods (preferences verbally are expressed to a non-dimensional utility function and the individual utility functions are combined into a utility function) and other methods. [12]

\section{Preference Template of D-Robots}

Preference of D-Robots and trade-off. Preference will be used for D-Robots to make decision when either mathematical equations or high level utility functions are not available.

A D-Robot's preference is situated based on its environment and depends on certain psychological characters including thinking style, decision making style, degree of risk-aversions, working style etc.. Many methods can be used for constructing preferences. An easy way to construct preferences is assigning values to preference for solution A over solution B. The preference of a D-Robot can be assigned or learnt from a human engineer through design cases.

Two D-Robots may have same strategy and rules but different preferences to solve same design problems. A group of D-Robots are assigned different decision making styles can reduce design defects and promote novel design. A D-Robot makes decision on better solutions and a group of D-Robots from different domains trade-off between their solutions. Therefore, the decision making is mostly multiobjective optimization or multidisciplinary optimization problem. 
Utility functions of D-Robots. The way to construct utility functions of D-Robots depends on the design tasks they execute. Mathematic objective functions and constraints will be established when numerical relationship can be established, e.g. in detail design stage. High level utility functions will be constructed when only discrete rules are available, e.g. in the conceptual design stage. In most cases, two kinds of utility functions may be required even for detail design because mathematical models can only encapsulate part of attributes and it is not uncommon that no feasible solution can be produced for a multiobjective optimization problem.

The basic idea to construct high level utility functions is assigning reward or utility values to various outcomes of a decision-making process. For example, the reward/utility could indicate the amount of components introduced to achieve a specific goal or function or behavior, the resource availability, reuse level of resource, noise level, pollution level, confidence in the solution etc. If the reward/utility values have been determined in a way consistent with the axioms, there exists a utility function $\mathrm{U}$.

Template of Preference. Based on discussion in previous paragraphs, a preference template can be formulated.

Preferences of D-Robots can be classified into several groups: Domain preference, Thinking style preference, Decision making style, Degree of risk-aversion and Working style. Domain preference refers to professional domain-based preference. For example, a D-Robot may have a mechanical preference to use a mechanism to transform a rotation motion to a reciprocating movement, such as cam mechanism, linkage mechanisms or a rack and pinion gears, while another D-Robot may have a control preference that prefers to use a linear motor. Hence, a mechanical domain D-Robot "knows" mechanical design would prefer to use mechanisms and mechanical components to achieve a specific motion control, while a control domain D-Robot "knows" control design would prefer to use electronic hardware to achieve same task. Different prefernces may lead to different solution seraching methods. For example, two D-Robots have different decision thinking styles and risk aversion styles. D-Robot A likes to use existing components, while the other prefers to search optimal shapes and configuration, or novel solutions. The preference template is illustrated in Figure 1.

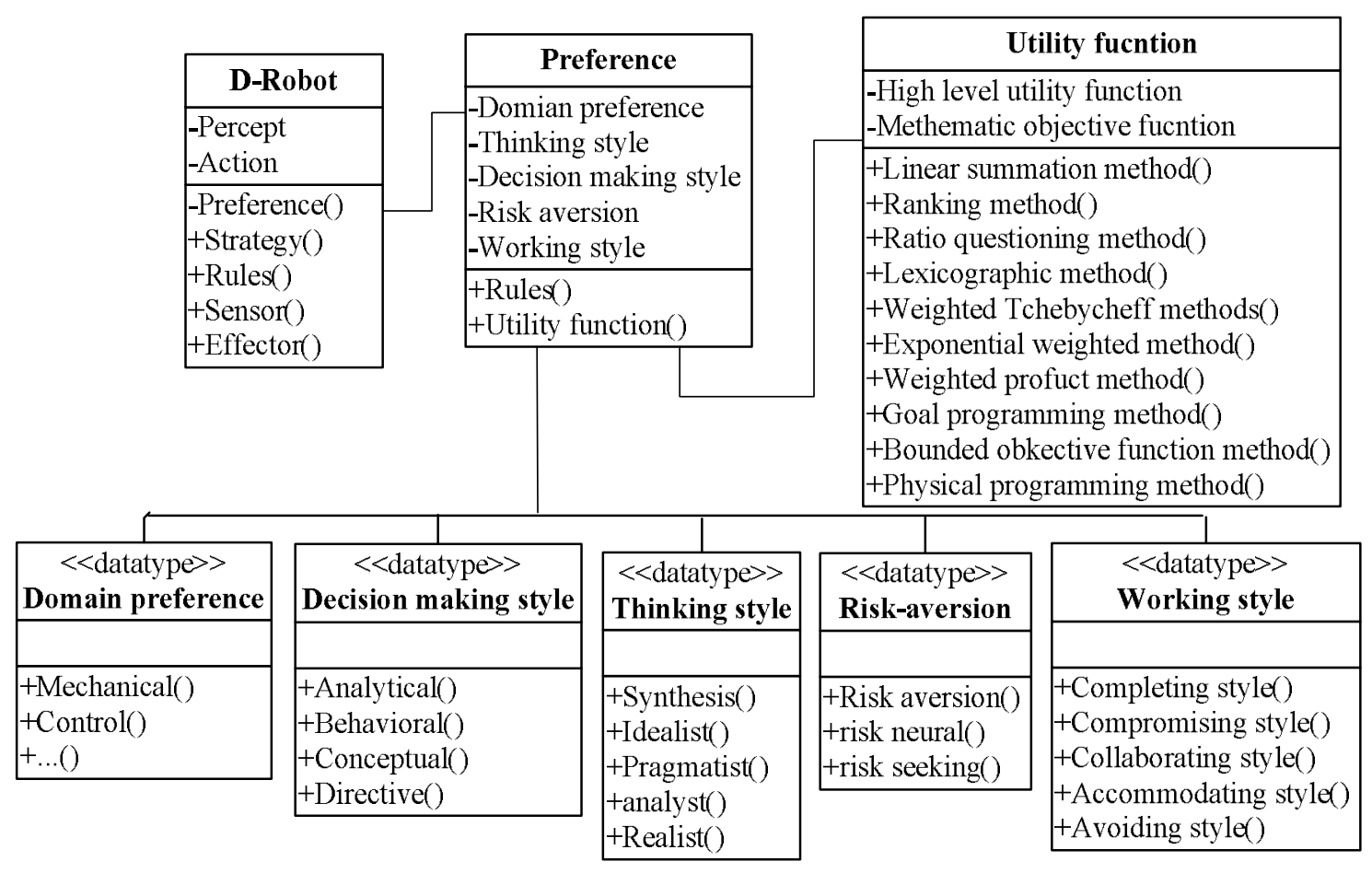

Figure 1 Preference template 


\section{Conclusion and discussion}

Toolkits that assist engineers rather than substitute them in conceiving and analysis are practical tools to design products, particularly for engineering products. A group of D-Robots collaborate together to reduce design defects and to promote the novelty of a product. The main difference between D-Robots and agents is that D-Robots have preferences, are capable of creating situated action plans and execute the plans. A preference template is constructed, including six groups of preferences: Domain preference, Thinking style preference, Decision making style, Degree of risk-aversion and Working style, as well as decision rules for different preference groups and method to construct utility functions. The future work is to develop algorithms for constructing preference of D-Robots.

\section{Acknowledgements}

This research is partly supported by the National Natural Science Foundation of China (51175284) and Education Committee of Beijing Municipality (grant No. SQKM201211232002).

\section{References}

[1] J. van der Geer, J.A.J. Hanraads, R.A. Lupton, The art of writing a scientific article, J. Sci. Commun. 163 (2000) 51-59.

[1] Y. Hou, L. Ji, Role of D-Robots in Designing, Advanced Materials Research. 694-697 (2013), 1717-1721.

[2] J. Broome, Reasoning with preferences? in S. Olsaretti (EDs), Preferences and Well-Being, Cambridge University Press, 2006, 183-208.

[3] European inland recreational fisheries, Methodologies for assessing socio-economic benefits of European inland recreational fisheries, Food and agriculture organization of the united nations, Ankara, 2010.

[4] S. Lichtenstein, P. Slavic, The Construction of Preference: An Overview, Cambridge University Press, 2006.

[5] A.F. Harrision, R.M. Bramson, The Art of Thinking, Berkeley Book, New York, 1984. http://comptalk.fiu.edu/thinking_styles.htm.

[6] A. J. Rowe, R. O. Mason, Managing with style: A guide to understanding, assessing, and improving decision making. San Francisco, California: Jossey Bass. 1987.

[7] A. J., Rowe, J. D. \& Boulgarides, Managerial Decision Making: A Guide to Successful Business Decisions. New York: McMillan, 1992.

[8] (http://en.wikipedia.org/wiki/Risk_aversion

[9] Risk Aversion, Chapter 2, http://www.public.asu.edu/ kirkwood/DAStuff/decisiontrees /DecisionTreePrimer-2.pdf.

[10] D. Van Bossuyta, C. Hoylea, I.Y. Tumera, A. Donga, Risk attitudes in risk-based design: Considering risk attitude using utility theory in risk-based design, Artificial Intelligence for Engineering Design, Analysis and Manufacturing, 26 (4), 2012, 393-406.

[11] http://www.dougsguides.com/context

[12] R.T. Marler, J.S. Arora, Survey of multi-objective optimization methods for engineering, Struct Multidisc Optim, 26, 2004, 369-395. 